\title{
GEOPOLÍTICA CRÍTICA: ALCANCES, LIIMITES Y APORTES PARA LOS ESTUDIOS INTERNACIONALES EN SUDAMÉRICA
}

\author{
CRITICAL GEOPOLITICS: SCOPE, LIMITS \\ AND CONTRIBUTIONS TO INTERNATIONAL \\ STUDIES IN SOUTH AMERICA
}

\section{LA GÉOPOLITIQUE CRITIQUE: SA PORTÉE, SES LIMITES ET SA CONTRIBUTION AUX ÉTUDES INTERNATIONALES EN AMÉRIQUE DU SUD}

\author{
Lester Cabrera Toledo \\ FLACSO Ecuador \\ lecabrerafl@flacso.edu.ec
}

\begin{abstract}
Resumen: Este artículo tiene por objetivo discutir, en términos epistemológicos, teóricos y metodológicos, el concepto de geopolítica crítica y su vinculación con los estudios internacionales. Así, se analizarán los alcances, límites y aportes del concepto, comparando entre los postulados clásicos y críticos de la geopolítica, y estableciendo un vínculo con la realidad sudamericana, región en la que el conocimiento geopolítico ha tenido un desarrollo relevante. Se concluye que es necesaria la vinculación entre la noción clásica y crítica de la geopolítica, con el objetivo de lograr una actualización de los postulados de la disciplina que priman en Sudamérica.
\end{abstract}

Palabras clave: geopolítica, discurso, Sudamérica, territorio, metodología.

Aвstract: The purpose of this paper is to discuss the concept of critical geopolitics in epistemological, theoretical and methodological terms, and its connection with international studies. It will analyze the scope, limits and contributions of the concept, comparing the classic and critical postulates of geopolitics, and establishing a link with the reality of South America, a region where geopolitical knowledge has seen significant development. It concludes that the connection between the classic and critical notion of geopolitics is necessary, in order to bring about an updating of the postulates of the discipline that still dominate in South America. 
Keywords: geopolitics, discourse, South America, territory, methodology.

\section{Traducción de Gonzalo Celorio Morayta}

RÉSumÉ: L'article s'interroge -en termes épistémologiques, théoriques et méthodologiques- sur la géopolitique critique et ses liens avec les études internationales. Pour analyser la portée, les limites et la contribution de cette discipline, on compare les principes classique et critique de la géopolitique, et on les met en rapport avec la situation de l'Amérique du Sud, qui est un territoire où les études géopolitiques se sont développées d'une façon considérable. On en tire la conclusion qu'il convient de lier les deux approches de la géopolitique, afin de mettre à jour les propositions de cette discipline qui prédominent dans la région.

Mots clefs: Géopolitique, discours, Amérique du Sud, territoire, méthodologie.

Traducción de BERNARdo Mabire

Fecha de recepción: octubre de 2018

Fecha de aceptación: enero de 2019 


\section{INTRODUCCIÓN}

- Cuáles son los principales problemas y aportes que se ponen de manifiesto en el concepto de geopolítica crí- tica? ¿Cómo se ha manifestado la geopolítica crítica en Sudamérica? El presente trabajo tiene como principal objetivo responder a las interrogantes planteadas, pero, sin perjuicio de un posterior desarrollo, resulta conveniente generar respuestas simples a modo introductorio. La geopolítica crítica nace como respuesta a los postulados "clásicos" de aquella área del conocimiento, especialmente teniendo en consideración las prácticas de los responsables de tomar decisiones y del contexto sobre el cual se establecían los postulados geopolíticos. En este plano, la postura crítica de la geopolítica, deconstruye los postulados clásicos, desde un punto de vista tanto de su puesta en práctica, como de los aspectos que influyen en su elaboración, aportando otras perspectivas analíticas para observar un determinado fenómeno social, preferentemente vinculado al territorio y sus representaciones. No obstante lo mencionado, este enfoque posee limitaciones en su entendimiento, su método de aplicación y su vinculación real con la geopolítica. Así, es posible dilucidar que, en múltiples puntos, la geopolítica crítica pasa de una revisión de los postulados geopolíticos clásicos a un análisis de discurso, dejando de lado factores clave para el análisis geopolítico, como lo es la propia condición geográfica. Al mismo tiempo, la geopolítica crítica establece patrones de análisis que ayudan a explicar fenómenos y procesos sociales, especialmente en el plano internacional, abriendo el camino a otras perspectivas disciplinarias que complementan el conocimiento y la explicación geopolítica de los fenómenos territoriales.

Con respecto a la segunda interrogante, el desarrollo del conocimiento geopolítico en Sudamérica ha tenido un fuerte auge en comparación con otras zonas geográficas, especialmente en contextos históricos donde la disciplina era considerada una "ciencia nazi", producto de su utilización para 
justificar la expansión territorial de la Alemania nazi. Así, la geopolítica en la región fue desarrollada especialmente por los miembros de las Fuerzas Armadas, generando al mismo tiempo sus propios postulados y lineamientos, tomando en consideración las realidades geográficas y el posicionamiento regional. Sin embargo, tales postulados, incluso una vez finalizado el conflicto bipolar, han mantenido la noción epistemológica de las nociones clásicas, disminuyendo la relevancia de las corrientes más contemporáneas de la geopolítica, tanto por el desconocimiento de éstas, como por el hecho de que el conocimiento geopolítico ha permanecido vinculado al estamento militar.

El objeto de estudio es analizar, desde una perspectiva conceptual, las limitaciones, alcances y aportes que se avizoran en la aplicación del análisis que aporta la geopolítica crítica. En este sentido, se comienza desde una perspectiva comparada, describiendo los principales elementos que "critica" la geopolítica crítica respecto a los postulados clásicos, para luego advertir y analizar las eventuales limitaciones y alcances en torno al concepto en sí, decantando en aquellos elementos que permiten generar nuevas perspectivas de explicación a fenómenos tanto locales como internacionales. Uno de los aspectos que llama la atención en relación a esto es que el mismo objeto de análisis de la geopolítica crítica sea difuso, lo que a su vez tiene como consecuencia una mayor amplitud al establecer la definición de geopolítica crítica e, incluso de la misma relevancia, delimitirla frente a lo que no es.

El material estudiado da cuenta de la evolución en la forma en que se entiende la separación de las geopolíticas mencionadas, como también de un aumento progresivo en el debate sobre los elementos que forman el conocimiento en geopolítica crítica. No obstante, si bien los tópicos sobre los cuales la geopolítica crítica se centra han aumentado, también lo han hecho las perspectivas difusas. Respecto a éstas, cabe mencionar que no aportan propuesta alguna, ni teórica ni metodológica, ni ayudan a mejorar el nivel de análisis o a proponer nuevas formas de entender las cuestiones analizadas. 
Entre las propuestas novedosas de la geopolítica crítica, destacan dos. En primer lugar, se observa que la geopolítica crítica pretende establecer un ordenamiento y clasificación de las representaciones geopolíticas, a través del propio origen de los discursos asociados a éstas. Con ello, no solamente se vislumbra una capacidad de generar nuevo conocimiento geopolítico, sino que también se hacen hallazgos sobre eventuales contradicciones en los discursos que construyen realidades sociales. Y, en segundo lugar, producto de la propia representación en el imaginario geopolítico, la perspectiva que entregan los denominados "códigos geopolíticos" ofrece nuevos esquemas de socialización en lo relativo a las amenazas e intereses de los países en general, como de los responsables de tomar decisiones, en particular, al momento de hacer referencia a los intereses internacionales de los Estados.

Se concluye que, si bien la geopolítica crítica es un campo que experimenta un aumento progresivo respecto a su utilización, ello aún no es debidamente comprendido, producto del desconocimiento prevaleciente de la disciplina, de las herramientas metodológicas difusas y de una perspectiva teórica cuya dirección aún no es clara. Pero el desafío radica tanto en el hecho de poder efectuar un análisis geopolítico, sin dejar de lado aquellos aspectos centrales de la disciplina geopolítica, como el territorio y el espacio, y vincular los preceptos tanto clásicos y críticos, como una forma de mejorar y enriquecer el conocimiento y las inferencias que puedan efectuarse, especialmente desde la óptica regional sudamericana, donde el conocimiento geopolítico sigue teniendo gran influencia en la formulación de las políticas exterior y de defensa.

\section{Geopolítica CRítica como Respuesta a "Lo ClÁsico"}

Cuando se tiene una perspectiva en torno a lo que implica el concepto "geopolítica", por lo general se establece una aso- 
ciación que es vaga, difusa y compleja. ${ }^{1}$ En primer lugar, ha sido subutilizada para hacer referencia a fenómenos que, sin ser necesariamente de naturaleza geopolíticos, se les atribuye tal característica, como una búsqueda para explicar los factores que ocasionan el problema. ${ }^{2}$ La concepción difusa de la geopolítica se basa en el hecho de que, si bien se tienen múltiples puntos de vista sobre la disciplina, también hay una gran cantidad de definiciones que enfatizan diferentes elementos y perspectivas, ${ }^{3}$ sin encontrar, necesariamente, puntos de conflicto al respecto. $Y$, finalmente, la complejidad de la geopolítica es una consecuencia de lo mencionado; es decir, hay diferentes escuelas de pensamiento que generan una mayor complejidad en la definición sobre lo que es, y lo que no es, geopolítica. ${ }^{4}$

De acuerdo con múltiples autores, ${ }^{5}$ la principal diferenciación, considerando elementos epistémicos y teóricos, de-

1 Phil Kelly, "A Critique of Critical Geopolitics", Geopolitics, vol. 11 (2006), pp. 24-53.

2 John Agnew, Geopolitics: Re-visioning World Politics, Londres, Routledge, 2003; Gearoid O'Tuathail, Critical Geopolitics. The Politics of Writing Global Space, Londres, Routledge, 1996.

3 Martin Müller, "Reconsidering the concept of discourse for the field of critical geopolitics: Towards discourse as language and practice", Political Geography, núm. 27 (2008), pp. 322-338; Colin Flint, Introduction to Geopolitics, Londres, Routledge, 2006; Peter Taylor y Colin Flint, Geografía política. Economía mundo, Estado-nación y localidad, Madrid, Trama Editorial, 2002; Simon Dalby, Creating the Second Cold War. The Discourse of Politics, Londres, Pinter Publishers, 1990.

4 Yves Lacoste, Geopolítica. La larga historia de hoy, Madrid, Síntesis, 2008; Phil Kelly, Classical Geopolitics. A New Analytical Model, Stanford, University Press, 2016.

5 Phil Kelly, op. cit. 2006; Gearoid O'Tuathail, “Thinking Critically about Geopolitics”, en Gearoid O'Tuathail, Simon Dalby y Paul Routledge (eds.), The Geopolitics Reader, Londres, Routledge, 2006, pp. 1-14; David Atkinson y Klaus Dodds, "Introduction to geopolitical traditions: a century of geopolitical thought", en Klaus Dodds y David Atkinson (eds.), Geopolitical Traditions. A century of geopolitical thought, Nueva York, Routledge, 2000, pp. 1-25; Geoffrey Sloan y Colin Gray, "Why geopolitics?", Journal of Strategic Studies, vol. 22 (1999), pp. 1-11. 
viene de la separación entre la denominada geopolítica "clásica" y la "crítica". La geopolítica clásica se denomina así por medio de los planteamientos vinculados, fundamentalmente, a la epistemología alemana de comienzos del siglo xx, donde se entendía el Estado como un organismo vivo, al tiempo que se le señalaba como la principal unidad de análisis. ${ }^{6}$ Como complemento de lo anterior, resulta adecuado presentar algunas definiciones al respecto. Para Rudolf Kjëllen, el autor que por primera vez acuñó el concepto, la "geopolítica es la ciencia que concibe el Estado como un organismo geográfico o como un fenómeno en el espacio”. Otra definición desde la perspectiva clásica es la de Karl Haushofer: "es la base científica del arte de la actuación política en la lucha a vida o muerte de los organismos estatales por el espacio vital". ${ }^{7}$

Un análisis de las anteriores definiciones aporta un panorama sobre el que se vincula la noción clásica de la geopolítica con la teoría realista de los estudios internacionales, identificando al Estado como la principal unidad de análisis. A lo anterior debe añadirse, necesariamente, una postura en torno a que el razonamiento geopolítico se efectuaba sobre la base de una estructura darwiniana, en la que el Estado se encontraba en permanente lucha por su desarrollo y supervivencia. Aquello se interpretaba como una forma de análisis basado en un juego de "suma cero" entre los países, en que la vinculación geográfica y el espacio se establecían como factores relevantes para la obtención de objetivos, especialmente a nivel internacional, siendo éste uno de los preceptos básicos de la concepción teórica realista. Además, la perspectiva de "suma cero" se trasplantabla al plano territorial, donde una posible ganancia o pérdida de este elemento significa, en el razonamiento geopolítico de la época, un

${ }^{6}$ Yves Lacoste, "Las etapas de la geopolítica”, en Leopoldo González Aguayo (edit.), Cuaderno de Trabajo. Antología. Los principales autores de las escuelas de la geopolítica en el mundo, México, UnAM, 2011, pp. 11-22; Gearoid O'Tuathail, op. cit., 1996.

7 Jorge Atencio, ¿Qué es la geopolítica?, Buenos Aires, Editorial Pleamar, 1968, pp. 23-25. 
cambio en el equilibrio de poder en términos absolutos. ${ }^{8}$ Incluso para la noción clásica esta perspectiva se considera un fundamento de la caída de imperios u otras formaciones políticas a lo largo de la historia. ${ }^{9}$

Otra de las cuestiones que se destacan en la visión clásica es la concepción ideológica subyacente que se tiene de ésta. Pese a que la geopolítica se planteó para entender el comportamiento de los países, lo cierto es que los diferentes "proyectos geopolíticos" o "teorías geopolíticas" que se esbozaron, especialmente en la primera mitad del siglo $\mathrm{xx}$, eran parte del posicionamiento de determinados Estados en el ámbito internacional. ${ }^{10}$ Por ese motivo, las "teorías geopolíticas clásicas" pueden considerarse como proyectos en el ámbito de la política exterior de una serie de países, que buscaban tener un posicionamiento hegemónico en el sistema regional e internacional imperante. Por esta razón, para determinados autores la geopolítica clásica puede considerarse una parte sustancial de un proyecto hegemónico e imperialista. ${ }^{11}$

Como una manera de responder a aquellos fenómenos e interpretaciones, en conjunto con el contexto académico imperante sobre la propia censura de la geopolítica clásica, surge la geopolítica crítica. Y al igual que la perspectiva clásica, la visión crítica puede interpretarse desde diferentes puntos de vista. Así, para Klaus Dodds, Merje Kuus y Joanne Sharp, la mencionada disciplina "es un subcampo de la geografía humana, la cual investiga las aseveraciones y supuestos geo-

${ }^{8}$ Phil Kelly, op. cit., 2016; Saul Cohen, Geopolitics. The Geography of International Relations, Nueva York, Rownman \& Littlefield, 2015; Klaus Dodds, Geopolitics: A Very Short Introduction, Nueva York, Oxford University Press, 2007.

${ }^{9}$ Joan Nogué y Joan Ruffí, Geopolítica, identidad y globalización, Barcelona, Ariel Geografía, 2001.

${ }^{10}$ John Agnew, op. cit., 2003; Gearoid O'Tuathail, op. cit., 1996.

${ }^{11}$ David Atkinson y Klaus Dodds, op. cit:; Gearoid O'Tuathail y Simon Dalby, "Introduction: Rethinking geopolitics: towards a critical geopolitics", en Gearoid O'Tuathail y Simon Dalby (eds.), Rethinking Geopolitics, Londres, Routledge, 1998, pp. 1-15. 
gráficos que se establecen en la política mundial. Para realizar aquello, examina las prácticas sobre las cuales los actores políticos 'espacializan' la política internacional, al tiempo que representan 'el mundo' caracterizado por lugares particulares”. ${ }^{12}$ Otra definición es la de Alison Mountz, al señalar que la geopolítica crítica "es el campo que examina la producción discursiva de 'los otros' y las formas que aquella producción del conocimiento se informa en las relaciones internacionales, especialmente los vinculados a los proyectos imperiales y coloniales". ${ }^{13}$ Finalmente, Heriberto Cairo establece que la geopolítica crítica es una disciplina auxiliar al Estado, y la define como "una práctica discursiva por la cual diversos grupos de responsables de tomar decisiones de gobierno (intellectuals of statecraft) 'espacializan' la política internacional para representar un 'mundo' caracterizado por tipos determinados de lugares, gentes y relatos". ${ }^{14}$

De acuerdo con la definiciones proporcionadas, la geopolítica crítica se posiciona como una respuesta necesaria a lo esbozado por los autores clásicos, en especial como consecuencia de la deslegitimación de la disciplina producto de la vinculación con la ideología nazi. ${ }^{15}$ La perspectiva crítica, que toma sus herramientas teóricas desde el posestructuralismo y las visiones "reflectivistas" o "interpretativistas" de los estudios internacionales, ${ }^{16}$ posiciona el discurso geopolítico como una realidad social construida y que, por ende, puede

12 Klaus Dodds, Merje Kuus y Joanne Sharp, "Introduction: Geopolitics and its Critics", en Klaus Dodds, Merje Kuus y Joanne Sharp (eds.), The Ashgate Research Companion to Critical Geopolitics, Londres, Ashgate Publishing, 2013, pp. 1-14.

${ }^{13}$ Alison Mountz, "The Other", en Carolyn Gallaher et al. (eds.), Key Concepts in Political Geography, Londres, SAGE, 2009, pp. 328-338.

${ }^{14}$ Heriberto Cairo, "Re-pensando la geopolítica: la renovación de la disciplina y las aportaciones de John A. Agnew" (Prólogo), en John Agnew, Geopolítica. Una re-visión de la política mundial, Madrid, Trama Editorial, 2005, pp. 9-16.

${ }^{15}$ Yves Lacoste, op. cit., 2008; Joan Nogué y Joan Ruffí, op. cit.

16 Klaus Dodds, Global Geopolitics. A Critical Introduction, Londres, Pearson Prentice Hall, 2005. 
analizarse por sí sola. Además, coloca en un sitial de relevancia para el análisis factores como el contexto político, que encierra la generación del discurso geopolítico, como una forma de apreciar nociones como el imperialismo y la hegemonía, en especial en el plano de las políticas exteriores esgrimidas por los países. ${ }^{17}$

Desde la visión crítica de la geopolítica, el pensamiento clásico no solamente es absoluto, sino limitado, principalmente a la hora de concebir su unidad de análisis. Si bien es cierto que el Estado es relevante, desde la geopolítica crítica éste no se interpreta como un todo absoluto y homogéneo. El pensamiento crítico en geopolítica propugna elementos que van desde la necesidad de incorporar las identidades como factor diferenciador en las sociedades, nociones económicas que grafiquen y expliquen una eventual "categorización" de las sociedades e, incluso, las formas de razonamiento que se observan entre los responsables de tomar decisiones. ${ }^{18}$ Desde este punto de vista, la geopolítica crítica toma una posición en torno a los discursos derivados del pensamiento clásico, que considera como una declaración de intereses; es decir, no adopta un posicionamiento o razonamiento neutral. ${ }^{19}$ En esencia, y siempre desde la perspectiva crítica, la geopolítica clásica encapsula, de acuerdo con los responsables de tomar decisiones en política exterior, el deseo de mantener un determinado orden nacional y, especialmente, internacional, con el fin de conservar o ganar una posición de superioridad con respecto a "los otros". ${ }^{20}$ Esta conducta, en los años en que los clásicos esgrimieron sus lineamientos

17 Gearoid O'Tuathail y John Agnew, "Geopolitics and discourse: Practical geopolitical reasoning in American Foreign Policy", Political Geography, vol. 11, (1992), pp. 190-204; Simon Dalby, op. cit., 1990.

18 Terrence Haverluk, Kevin Beauchemin y Brandon Mueller, "The Three Critical Flaws of Critical Geopolitics: Towards a Neo-Classical Geopolitics”, Geopolitics, núm. 19 (2014), pp. 19-39.

${ }^{19}$ Klaus Dodds, Merje Kuus y Joanne Sharp, op. cit.

${ }^{20}$ Carl Dahlman, "Geopolitics", en Carolyn Gallaher et. al. (eds.), Key Concepts in Political Geography, Londres, SAGE, 2009, pp. 87-98. 
geopolíticos, se corresponde con declaraciones y acciones de diversos Estados, como la Gran Bretaña de Mackinder, o la Alemania de Haushofer. ${ }^{21}$ Por lo tanto, la geopolítica clásica también se interpreta desde la perspectiva crítica como un instrumento que justifica medidas imperiales y hegemónicas en el ámbito internacional.

Un aspecto relevante en el campo de estudio de la geopolítica crítica responde al hecho de que, si bien ésta se ha considerado, cada vez más, en los análisis que pueden efectuarse, también es cierto que ha generado resistencias entre la propia comunidad epistémica, ${ }^{22}$ como también entre una serie de autores quienes, a su vez, critican sus postulados, fundamentalmente por disminuir el valor geopolítico de los autores clásicos. No obstante, al mismo tiempo se reconoce que, pese a las perspectivas contrarias, la geopolítica crítica ayuda a delimitar el conocimiento geopolítico, en especial cuando el concepto de geopolítica como tal se ha masificado, fundamentalmente en los medios de comunicación. ${ }^{23} \mathrm{Al}$ respecto, y a favor de los aportes de la geopolítica crítica, señalan Dodds, Kuus y Sharp que "reconociendo que muchos de los así llamados escritores geopolíticos estaban movilizando una comprensión simplista de los lugares y los espacios, la geopolítica crítica desafió esas preocupaciones, destacando por ejemplo la capacidad de recuperación de los hilos del pensamiento geopolítico como imperialista, nacionalista y racista”. ${ }^{24}$

${ }^{21}$ Gerry Kearns, "Imperial Geopolitics", en John Agnew, Katharyne Mitchell y Gearoid O'Tuathail (eds.), A Companion to Political Geography, Nueva York, Blackwell Publishing, 2003, pp. 173-186.

${ }^{22}$ Simon Dalby, "Imperialism, Domination, Culture: The Continued Relevance of Critical Geopolitics", Geopolitics, vol. 13, núm. 3 (2008), pp. 413-436; Peter Taylor, "Geopolitics, Political Geography and Social Science", en Klaus Dodds y David Atkinson (eds.), Geopolitical Traditions. A century of geopolitical thought, Nueva York, Routledge, 2000, pp.375-379.

${ }^{23}$ Gerry Kearns, op. cit.

${ }^{24}$ Klaus Dodds, Merje Kuus y Joanne Sharp, op. cit., p. 5. 
No obstante, los problemas y posiciones encontradas respecto a la geopolítica crítica van más allá del punto de vista que se adopta con respecto a los postulados clásicos. Así, es claro que las principales problemáticas se relacionan con el valor del discurso como un absoluto, la vinculación de los análisis con el territorio y la metodología con la cual opera.

\section{EL DISCURSO, LA TERRITORIALIDAD Y EL MÉTODO}

\section{COMO LIMITANTES}

La conceptualización de la geopolítica crítica, pese a que incluya una serie de elementos que evidencia aspectos comunes, también refleja una complejidad en lo relativo a la amplitud del concepto como tal. Un ejemplo de ello es el propio objeto de estudio sobre el que trabaja la geopolítica crítica. Cuando se efectúa un análisis sobre la base de la disciplina mencionada, el objeto central es, en la mayoría de los casos, difuso. Así, y como bien lo señala Dahlman, la geopolítica crítica, si se toma desde su base teórica posestructuralista, carece de un método único cuando explica un determinado tópico ${ }^{25} \mathrm{e}$, incluso, en este punto los autores no mencionan que exista un objeto de estudio delimitado, como sí lo tiene el enfoque clásico. ${ }^{26}$ En consecuencia, la geopolítica crítica no postula "leyes" o "teorías" que traten de comprender determinados fenómenos. Si bien esto se entiende por la propia concepción epistémica y ontológica de la geopolítica crítica, también es cierto que esta disciplina tiene frente a sí el desafío de construir y desarrollar su propio corpus teórico y analítico. Sin embargo, uno de las pocas cuestiones en las que es posible encontrar un aspecto delimitador es en torno a las perspectivas que se enfocan en desestimar las narrativas vinculadas al poder estatal, en sus diferentes niveles de acción. ${ }^{27}$

${ }^{25}$ Carl Dahlman, op. cit.

${ }^{26}$ Klaus Dodds, op. cit., 2007; Gearoid O’Tuathail, op. cit., 1996.

${ }^{27}$ Carl Dahlman, op. cit. 
La comprensión y el análisis del objeto de estudio para la geopolítica crítica, al escapar de la única unidad de análisis que postulaba la geopolítica clásica, pierde claridad. Por ello, la disciplina necesita otras herramientas para efectuar sus planteamientos. Es así que, y en línea con lo planteado por Martin Müller, el análisis de discurso, especialmente como insumo para el eventual logro de los objetivos que la disciplina propugna, se aprecia como un aspecto sólidamente vinculado a la geopolítica crítica. ${ }^{28}$ No obstante, pese a que el análisis de discurso se convierte en el núcleo de los eventuales lineamientos que se deduzcan de un fenómeno o proceso observado, también se aprecia, en forma mayoritaria, un análisis en sí mismo. ${ }^{29} \mathrm{Al}$ enfocar el análisis de discurso como un método más que como una técnica, y sumando su concepción teórica posestructuralista o reflectivista, la geopolítica crítica se limitaría principalmente a los análisis narrativos; pese a ello, el principal problema no se aprecia en la forma, sino cuando busca responder a las preguntas sobre qué se estudia y para qué se estudia. En estricto rigor, el análisis de discurso se diferencia medianamente de la geopolítica crítica cuando se da una respuesta satisfactoria a una interrogante señalada. Incluso así, el análisis carece de un elemento clave para denominarse geopolítico. ${ }^{30}$

Al generar explicaciones e inferencias sobre la base de un análisis, como sería para el caso de la geopolítica crítica, tomando como referencia el discurso, se efectúa el error de perder la cualidad "geopolítica" como tal, haciendo del análisis del discurso un fin en sí mismo. En este plano, la presencia de una determinada característica territorial es lo que otorga la condición geopolítica. El territorio, para algunos autores, marca el vínculo con la geopolítica, más allá del hecho de que al realizar una desagregación del discurso pue-

28 Martin Müller, op cit.

${ }^{29}$ John Agnew, op. cit.; Gearoid O’Tuathail y John Agnew, op. cit.; Simon Dalby, op. cit., 1990.

${ }^{30}$ Martin Müller, op. cit. 
dan evidenciarse nociones de poder y hegemonía, cuya base lógicamente es política y no necesariamente territorial. ${ }^{31} \mathrm{La}$ geopolítica, en cualquiera de sus dos grandes vertientes, se representa como una influencia de poder; sin embargo, la diferencia reside principalmente en la ubicación, representación y manifestación misma del poder. Sin embargo, aquel poder, sin la unión con el territorio, carece de una configuración geográfica como tal y, por ende, de la cualidad geopolítica.

Existen una multiplicidad de análisis que vinculan el poder con el territorio en el ámbito de la geopolítica crítica, ${ }^{32}$ pero la comprensión del territorio, desde una perspectiva posestructuralista, no necesariamente refleja los intereses de poder que supone e, incluso, la propia concepción subjetiva del territorio da como resultado una percepción difusa de los elementos que se vinculan a éste, siempre en términos de poder. De esta manera, el discurso y el territorio, si bien pueden compartir importantes segmentos de análisis, pueden también no compartirlo, originando una desnaturalización de la concepción geopolítica. Ejemplo de ello son los análisis de procesos "geopolíticos" que poco o nada se relacionan con la territorialidad. ${ }^{33}$

De acuerdo con Phill Kelly, ${ }^{34}$ hay un elemento que resulta fundamental para entender la limitación territorial que establece la geopolítica crítica, y que se vincula directamente tanto con la forma de comprender los actuales procesos políticos, como con el hecho de situarse en un determinado punto de vista epistémico para comprender el territorio. Así, si

${ }^{31}$ Klaus Dodds, Merje Kuus y Joanne Sharp, op. cit.

32 John Agnew, op. cit.; Wolfgang Natter, "Geopolitics in Germany, 1919-1945”, en John Agnew, Katharyne Mitchell y Gearoid O'Tuathail (eds.), A Companion to Political Geography, Nueva York, Blackwell Publishing, 2003, pp. 187-203.

33 Paul Routledge, "Introduction: Anti-Geopolitics", en Gearoid O’Tuathail, Simon Dalby y Paul Routledge (eds.), The Geopolitics Reader, Londres, Routledge, 2006, pp. 245-255.

${ }^{34}$ Phil Kelly, op. cit., 2006. 
bien es cierto que existe una visión en torno a la "desterritorialización" de los espacios geográficos, ${ }^{35}$ también lo es que la geopolítica no puede concebirse como tal sin la propia noción del territorio. Sin embargo, el problema no radica en la composición del territorio en sí misma, sino en su comprensión. Con ello, la visión de "espacio" resulta más adecuada que la de territorio, incluso para referirse a las realidades que se visualizan en el interior del Estado. El espacio, para la geopolítica crítica, se acopla a una visión dinámica y en constante cambio, incorporando la necesidad de tener diferentes niveles de entendimiento de éste. Desde esta perspectiva, el discurso se convierte en la forma de interpretar el espacio, así como todas sus diferencias, en las que es posible establecer el posicionamiento propio de un grupo social, el ordenamiento territorial de una ciudad o un asentamiento urbano, o las diferencias entre ruralidad y urbanidad. ${ }^{36}$

De esta manera, es posible dilucidar que la visión de territorio es una perspectiva absoluta y poco adaptable a los cambios que traen consigo los movimientos sociales, incluso desde el plano internacional; mientras que la noción de espacio, si bien toca el proceso de "desterritorialización", se efectúa sobre una base totalmente contraria al posicionamiento de la geopolítica clásica, para la cual los elementos geográficos influyen, casi de manera irremediable, en la forma de concebir las orientaciones políticas y sociales.

Otra de las dificultades de la geopolítica crítica radica en la metodología que emplea para efectuar sus análisis. Al respecto, si bien es cierto que dentro del propio análisis del discurso es factible encontrar una buena cantidad de referencias sobre cómo realizarlo, lo cierto es que esa perspectiva, como bien se mencionó en su momento, no necesariamente se vincula con lo que puede ser un análisis de características geopo-

35 John Agnew y Stuart Corbridge, Mastering Space. Hegemony, territory and international political economy, Londres, Routledge, 1995.

36 Stephen Graham, "Introduction: Cities, Warfare, and States of Emergency", en Stephen Graham (edit.), Cities, War, and Terrorism. Towards an Urban Geopolitics, Nueva York, Blackwell Publishing, 2004, pp. 1-26. 
líticas. Pero, incluso más allá de esto, desde una visión teórica, la geopolítica crítica carece de un método claro y específico, por lo que debe valerse de otras perspectivas para desarrollar sus análisis. ${ }^{37}$ Los principales autores de la disciplina han tomado en consideración esta debilidad, y si bien no han establecido una metodología de análisis, sí han desarrollado una manera de comprender los discursos que debe tomar en cuenta la disciplina, en atención a su origen. ${ }^{38}$ Sin embargo, esto no puede concebirse como un método propio de la geopolítica crítica, sino como una sistematización en torno al objeto de estudio. Y en este aspectose establecen dos elementos clave: la primacía del discurso en cualquier análisis de la geopolítica crítica y la carencia metodológica de la disciplina, que se suple con otro tipo de herramientas.

Como se mencionó en su momento, el discurso ocupa un lugar clave para la comprensión de los fenómenos que se propone analizar la geopolítica crítica. Pero si bien ello puede considerarse como un elemento que ayuda a entender los aspectos y realidades subyacentes de los diferentes contextos, también es cierto que limita la propia perspectiva geopolítica, vinculándose en este punto con el aspecto territorial. Cuando el discurso se convierte en el centro del análisis, se pierde una noción importante de la territorialidad. Esto se explica por el hecho de que los elementos en torno al análisis del discurso no son necesariamente territoriales, lo que da lugar a que cualquier discurso, sin importar el objeto o problema que se analice, puede incorporarse como un fenómeno a considerar perteneciente a la geopolítica crítica, lo cual no es necesariamente eficiente.

Mientras que en el segundo aspecto, las herramientas que se proponen desde la geopolítica crítica tienen una base sobre el propio discurso, sin generar una diferencia sustan-

${ }^{37}$ Klaus Dodds, Merje Kuus y Joanne Sharp, op. cit.

${ }^{38}$ Simon Dalby, op. cit., 2008; Gearoid O'Tuathail, "Postmodern geopolitics? The modern geopolitical imagination and beyond", en Gearoid O'Tuathail y Simon Dalby (eds.), Rethinking Geopolitics, Londres, Routledge, 1998, pp. 16-38. 
cial entre lo que puede entenderse por análisis de discurso, por un lado, y geopolítica crítica, por otro. ${ }^{39}$ La diferencia se presenta cuando se define el objeto de investigación, a la vez que se vincula adecuadamente con una realidad territorial, la cual bien puede ser deconstruida a través de los preceptos de la geopolítica crítica, pero debe tenerse presente para, de esa forma, hablar de geopolítica como tal.

\section{LOS APORTES DE LA GEOPOLÍTICA CRÍTICA}

Pese a las diferentes limitantes señaladas de la geopolítica crítica, ello no es necesariamente reflejo de una cualidad negativa, sino de un límite; pero tal perspectiva también puede considerarse un desafío, en cuanto a la incorporación de aproximaciones sociales a fenómenos que se relacionan con el territorio. En este plano, la geopolítica crítica puede ser una herramienta adecuada cuando sea conveniente asociarse, en los estudios internacionales, con diferentes maneras de entender y explicar la forma en que los actores del sistema internacional se relacionan, e incluso el propio comportamiento de éstos.

El campo de estudio de la geopolítica crítica, si bien establece el discurso como uno de los elementos centrales en su eventual análisis, no solamente se enfoca en el discurso en sí, ya que considera aspectos de la representación de un espacio geográfico, como en el origen del propio discurso. Es así que se establecen diferencias sustanciales al intentar comprender un cambio en la forma de representar el territorio, como consecuencia de la multiplicidad de puntos de vista propuestos. ${ }^{40}$ Pero además, el núcleo central se basa en el origen de los discursos. ${ }^{41}$ A raíz de ello, el discurso geopolítico encontraría su importancia al conocer y comprender el

${ }^{39}$ Martin Müller, op. cit.

${ }^{40}$ Phil Kelly, op. cit., 2006.

${ }^{41}$ Martin Müller, op. cit. 
contexto en que se dio tal discurso, los elementos que eventualmente influyeron en su generación y los aspectos subyacentes, generalmente simbólicos, que se encuentran en éste.

Es así como -y de acuerdo con Gearoid O'Tuathail y Simon Dalby-, el discurso debe tener una clasificación para poder explicar aspectos tales como la apreciación de las fronteras, la representación del espacio territorial y las amenazas que eventualmente perciben las personas responsables de tomar decisiones de política exterior en el Estado. La clasificación propuesta por tales autores se vincula con el propio origen de los discursos, tomando en consideración la fuente de éstos, pero desde un punto de vista institucional, estableciendo tres divisiones: la geopolítica práctica, que se relaciona con los discursos emitidos por los líderes de Estado y la burocracia a cargo de la política exterior del país; la geopolítica formal, dada como consecuencia de los discursos que emanan de centros de estudios y universidades; y, finalmente, la geopolítica popular, presente en los discursos que se emiten a través de los medios masivos de comunicación. ${ }^{42}$

En este sentido, es posible señalar que existen tres formas de comprender la "imagen" y representación que manifiesta el discurso, como herramienta y como generador de patrones sociales, en la geopolítica popular, la geopolítica práctica y la geopolítica formal. La categorización de cada una de éstas se refleja en el siguiente diagrama.

Como puede observarse, la geopolítica formal se enfoca en la construcción de un imaginario social que deviene de los discursos de los centros de investigación, las universidades y cualquier organización con una directa vinculación con el medio académico-profesional. En este ámbito, el discurso se manifiesta fundamentalmente a través de publicaciones como revistas, libros, o incluso artículos de opinión, donde se observen las perspectivas geopolíticas de un autor o bien de una institución.

${ }^{42}$ Gearoid O’Tuathaul y Simon Dalby, op. cit. 
FigurA 1

La geopolítica crítica y sus orígenes discursivos

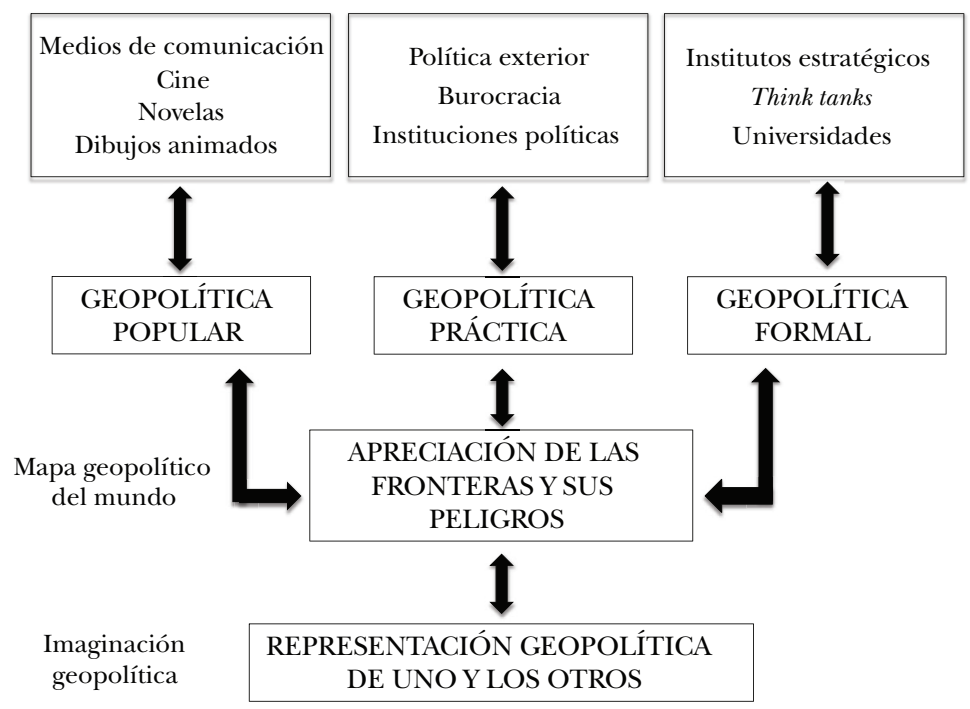

Fuente: Gearoid O'Tuathail y Simon Dalby, op. cit., p. 5.

Siguiendo el diagrama anterior, la geopolítica práctica se extrae de los discursos de las personas responsables de tomar decisiones, particularmente en el ámbito de la política exterior. Este punto es uno de los más complejos a tratar, si se considera la multiplicidad de actores que pudiesen estar involucrados en la generación de la política mencionada, así como por los múltiples canales que difunden una determinada imagen o discurso sobre un tema en cuestión. Sin embargo, las consideraciones en torno a las implicaciones estratégicas y territoriales son las que marcan una diferencia entre los discursos geopolíticos y de otro tipo, pese a que incluso con esa perspectiva resulte viable establecer apreciaciones geográficas desde una base económica o comercial. Finalmente, la geopolítica popular se da como consecuencia de los discursos derivados de los medios de comunicación masivos y de las expresiones culturales masivas como el cine o la televisión. En 
este ámbito, la dificultad se aprecia en la amplitud de fuentes $y$, por ende, en delimitar los elementos que subyacen al discurso popular, el cual, de acuerdo con los propios autores, esconde aspectos ideológicos que denotan una representación geopolítica que, en un primer momento, no es clara ni explícita. ${ }^{43}$

Otro de los elementos relevantes que integra la perspectiva de la geopolítica crítica es la generación del concepto "códigos geopolíticos". Esta perspectiva integra la concepción (o imágenes) de los responsables de tomar decisiones de política exterior de los Estados y la propia percepción sobre las amenazas que perciben, tomando en consideración la composición del sistema internacional como parte de un patrón de intereses. Así, para Colin Flint, los códigos geopolíticos pueden definirse como "la manera en que un país se orienta a sí mismo hacia el mundo"; ${ }^{44}$ otra definición plantea que son "un conjunto de supuestos estratégicos que elabora un gobierno sobre otros Estados para orientar su política exterior". ${ }^{45}$

Más allá de una eventual explicación sobre los elementos implícitos en esta categoría conceptual, los códigos geopolíticos "suponen la ponderación, según su evaluación estratégica y en tanto que amenazas potenciales, de lugares que están más allá de las fronteras del Estado". ${ }^{46}$ Este aspecto no es menor, ya que sitúa los códigos geopolíticos como parte de una interpretación mucho más amplia de lo que podría concebirse como una mera declaración de intenciones, desde el punto de vista de la política exterior. Esto se relaciona con las apreciaciones y percepciones que puede sostener un Estado, pero tomando en cuenta elementos que se relacionan con su seguridad, los que no necesariamente poseen la cualidad de provenir de un actor estatal. Por lo tanto, la con-

\footnotetext{
${ }^{43}$ Loc cit.

${ }^{44}$ Colin Flint, op. cit., p. 55.

45 Peter Taylor y Colin Flint, op. cit., p. 99.

${ }^{46}$ Loc cit.
} 
figuración de un código geopolítico supone cualidades que se asocian a la política exterior y directa o indirectamente, con la seguridad del Estado. ${ }^{47}$

Una de las cuestiones clave de la concepción de los códigos geopolíticos es el hecho de que todos los países lo tienen y se le ha denominado código geopolítico local. Así también, sólo algunos países, especialmente aquéllos con un grado relevante de influencia en sus entornos cercanos, tienen un código geopolítico de características regionales. Y, finalmente, algunos países tienen lo que los autores mencionados denominan un código geopolítico mundial. ${ }^{48}$ Esta clasificación cobra validez cuando un Estado se manifiesta en relación con temas locales, vecinales, regionales o mundiales, en consonancia con sus respectivos discursos, en los diferentes ámbitos que proponen tanto O'Tuathail como Dalby. Asimismo, según las propias expresiones oficiales y a través de los medios de comunicación es posible señalar que los códigos geopolíticos no son inmutables, sino que van evolucionando y cambiando con el contexto internacional y en la manera en que los responsables de tomar decisiones interpretan el escenario mencionado.

De esta manera, , desde un punto de vista teórico, las diferentes visiones de la geopolítica crítica comparten una serie de elementos en común que aportan una base para la mejor comprensión de los fenómenos que se desean analizar, a la vez que señala elementos o procesos que ayudarían a construir una perspectiva o abordaje propio de la geopolítica crítica. No obstante y sin perjuicio de lo mencionado, resulta adecuado establecer algunas precisiones. La primera gira en torno al grado de relación de los elementos teóricos que sustentan las posiciones de los diferentes autores, vinculados a la generación del conocimiento en la disciplina de la

${ }^{47}$ Lester Cabrera, "La vinculación entre geopolítica y seguridad: algunas apreciaciones conceptuales y teóricas”, URVIO, Revista Latinoamericana de Estudios de Seguridad, núm. 20 (2017), pp. 111-125.

${ }^{48}$ Peter Taylor y Colin Flint, op. cit., p. 101. 
geopolítica crítica. Esto permite clasificar las nociones que, si bien en un primer momento pueden parecer dispersas, tienen puentes conceptuales y teóricos que son parte de un campo de estudio singular. De este modo, es posible considerar el enfoque planteado por Gearoid O'Tuathail y Simón Dalby, en su trasfondo teórico, desde la perspectiva posestructuralista. Al mismo tiempo, los postulados geopolíticos que esgrimen autores como Peter Taylor y Colin Flint se sustentan en la economía política internacional, al considerar la noción de los códigos geopolíticos desde el punto de vista de Immanuel Wallerstein en lo que denomina el sistema-mundo y su estructura tripartita de centro, periferia y semiperiferia. ${ }^{49}$

$\mathrm{Al}$ respecto, cabe señalar que los postulados vinculados al posestructuralismo abogarían por la división de la geopolítica en práctica, formal y popular (mencionadas líneas arriba), y en todas éstas el discurso es el elemento central. Mientras que, por otro lado, la visión relacionada con la economía política internacional tiene como elementos conceptuales los denominados códigos geopolíticos, que establecen una "imagen" en torno a la propia proyección de los países hacia el mundo, los actores que lo componen y la evaluación de las amenazas y riesgos que presuponen para sus intereses. $\mathrm{Y}$ pese a las diferencias, ambas corrientes encuentran elementos en común que permiten una concatenación de supuestos y preceptos, ayudando así a la creación del campo de estudio. Por ejemplo, se muestra que el discurso y el concepto de "imagen" no solamente se relacionan en forma íntima, sino que además encuentran un soporte conceptual tanto en las diferentes clasificaciones de las geopolíticas como desde los propios códigos.

Lo anterior se entiende mejor si tomamos en cuenta que en ambas perspectivas, teóricas o conceptuales, el elemento central no pasaría a ser el discurso como tal, sino que una determinada "visión de mundo", lo que se traduce en el ob-

${ }^{49}$ Immanuel Wallerstein, Geopolítica y Geocultura. Ensayos sobre el moderno sistema mundial, Barcelona, Editorial Kairós, 2011. 
jeto de estudio de la geopolítica crítica, considerando las diferentes relaciones y vinculaciones que se suceden en el ámbito internacional. Por esta razón, la "visión del mundo" es el elemento que une ambas nociones teóricas. Incluso, es posible coincidir en aquello con planteamientos que propugnan por mantener el Estado como la principal unidad de análisis, incluso desde un punto de vista crítico de la geopolítica. ${ }^{50}$ Así, las vinculaciones mencionadas pueden expresarse en la siguiente figura:

FigurA 2

Vinculaciones teóricas y conceptuales de las diferentes corrientes de la geopolítica crítica

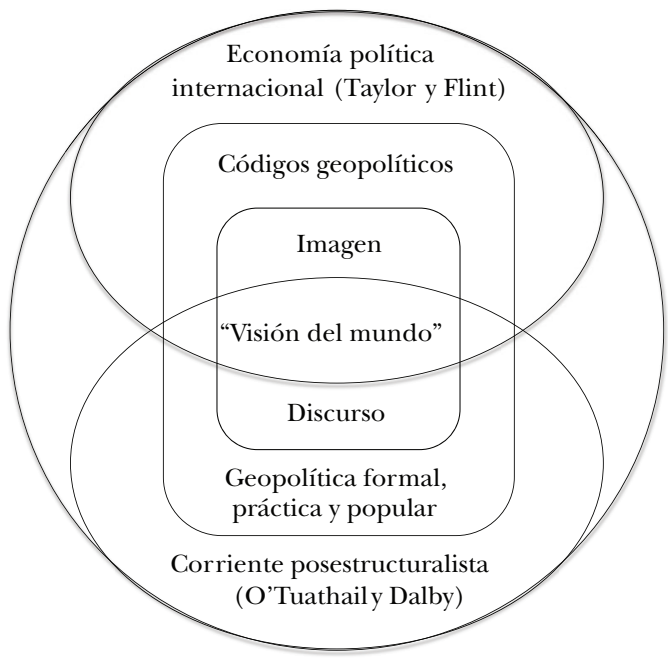

Fuente: elaboración propia.

Si bien esto último puede considerarse como parte de una propuesta en torno a la generación de los preceptos teóricos que sustentan la geopolítica crítica, aquello es digno de

${ }^{50}$ Klaus Dodds, op. cit., 2007. 
debate y de diversas observaciones. No obstante, en términos generales, los aportes de la geopolítica crítica pueden resumirse en dos grandes aspectos: la "revitalización" de la geopolítica como disciplina académica y la capacidad de explorar nuevos elementos y conceptos que aportan otros puntos de vista, más allá de los que generan los postulados clásicos, frente a fenómenos principalmente internacionales y regionales. Y es frente a aquella realidad en donde la región de América del Sur presenta una serie de procesos que evidencia la necesidad de debatir y evolucionar de acuerdo con la manera en que la geopolítica se presenta e interpreta, como campo de estudio.

\section{GEOPOLÍTICA Y SU VISIÓN CRÍTICA EN EL CONTEXTO SUDAMERICANO}

La geopolítica en Sudamérica ha sido una de las disciplinas más desarrolladas en el plano de las áreas del conocimiento que vinculan al Estado con su entorno internacional. ${ }^{51}$ Lo anterior, tanto en comparación con otras disciplinas similares, como por el hecho de que mientras en otras latitudes del mundo la geopolítica se consideraba un conocimiento "tabú" debido a su vinculación con el expansionismo de la Alemania nazi. En la región sudamericana se siguieron desarrollando preceptos y lineamientos geopolíticos de acuerdo con las cualidades de cada país. ${ }^{52}$ Es así como en algunos de ellos, la evolución del conocimiento geopolítico alcanzó un nivel alto, lo cual se reflejaba tanto en políticas como en preceptos conceptuales propios, tomando como casos de estudio principalmente las realidades de Brasil, Argentina y Chile, considerados

${ }^{51}$ Detlef Nolte y Leslie Wehner, "Geopolitics in Latin America, Old and New", en David Mares y Arie Kacowicz (eds.), Routledge Handbook of Latin American Security, Nueva York, Routledge, 2016, pp. 33-43.

${ }^{52}$ Phil Kelly, op. cit., 2016; Jonathan Barton, A Political Geography of Latin America, Nueva York, Routledge, 1997. 
incluso "escuelas geopolíticas". ${ }^{53}$ Sin embargo entender la expansión de esta rama del conocimiento, cuya raíz es eminentemente clásica, exige estudiar una serie de procesos que la región vivió durante el siglo $\mathrm{xx}$.

Uno de esos procesos se relaciona con la interpretación que se hizo de la geopolítica, especialmente en la pos Segunda Guerra Mundial, desde el punto de vista de su objeto de estudio. En este plano, se distingue que la noción clásica de la disciplina fue predominante en la región con la consecuencia de que el Estado fue el actor principal en los diferentes análisis relacionados con la geopolítica. Este punto es clave porque, como consecuencia de ese precepto, en la región se vinculó con fuerza la geopolítica con el realismo. ${ }^{54} \mathrm{Y}$ esto último se vio maximizado como producto de la interpretación y el desarrollo de la geopolítica, en especial en pleno conflicto bipolar, tomando en cuenta que fue el segmento militar el que mayormente buscó recurrir a la geopolítica como herramienta que le permitiera prever y analizar los posibles puntos de conflicto con otros países. ${ }^{55} \mathrm{Al}$ mismo tiempo, la geopolítica comenzó a tener una perspectiva nacional, estableciéndose como una disciplina que ayudaba a tomar decisiones en el plano de la política exterior de los Estados y, así, lograr o mantener sus intereses en el ámbito regional y en el entorno de países vecinos, lo cual se demostró especialmente cuando los gobiernos militares fueron la tónica en Sudamérica. ${ }^{56}$

No obstante, concebir el pensamiento y desarrollo de la geopolítica en Sudamérica como un todo es un error. Incluso en la forma en que ésta se concibió en un principio y luego se expandió, es posible encontrar diferencias sustan-

${ }^{53}$ John Child, "Geopolitical Thinking in Latin America", Latin American Research Review, núm. 14, vol. 2 (1979), pp. 89-111.

${ }^{54}$ Lester Cabrera, op. cit.

${ }^{55}$ Fredercik Nunn, Relaciones militares civiles sudamericanas en el siglo XXI. Sombras del pasado y formas de lo que vendrá, Santiago, Academia de Guerra del Ejército de Chile, 2011.

${ }^{56}$ Jonathan Barton, op. cit:; John Child, op. cit. 
ciales que ayudan a explicar las nociones de regiones e incluso los intereses de los países de manera particular. ${ }^{57}$ De esta manera, al analizar la evolución del conocimiento geopolítico en Sudamérica desde un punto de vista crítico, es posible mostrar dos grandes perspectivas: la que se deriva de la realidad del Cono Sur, y la lógica del pensamiento brasileño y uruguayo.

Uno de los puntos fuertes de la noción que se manifiesta del pensamiento geopolítico propio del Cono Sur deviene de una concepción realista de la geopolítica, en la cual los preceptos elaborados, especialmente por autores militares, buscaban presencia sobre determinados lugares territoriales, con el objetivo de lograr una mayor influencia y proyección de los Estados. ${ }^{58}$ En este plano, la geopolítica se interpretaba como un medio para que los países mantuvieran su territorio, aumentar su influencia y proyección y, tal vez lo más relevante, evitar el acceso de otro país a esas posiciones. Bajo aquella lógica, la geopolítica sirvió para justificar y argumentar conflictos, que tenían también un componente que se derivaba de la historia bilateral y, principalmente, de los conflictos bélicos vividos en el pasado. Pese a que señalaba la relevancia de la presencia de las instituciones del Estado en la extensión territorial de un país en particular, el foco de la geopolítica para los países de esta región se centraba en el plano internacional y de países vecinos. ${ }^{59}$

A diferencia de la concepción del Cono Sur, la perspectiva de Brasil y Uruguay presenta elementos que la acercan más a los postulados iniciales de la disciplina, considerando el punto de vista del creador del concepto como tal. En este sentido, la geopolítica se interpreta en su relación con el desarrollo de los países, en otras palabras, tiene una connota-

${ }^{57}$ Leslie Hepple, "South American Heartland: The Charcas, Latin American Geopolitics and Global Strategies", The Geographical Journal, vol. 170, núm. 4 (2004), pp. 359-367.

${ }^{58}$ Carlos Mierelles, Antología geopolítica de autores militares chilenos, Santiago, Centro de Estudios e Investigaciones Militares, 2000.

${ }^{59}$ Jorge Atencio, op, cit:; Carlos Mierelles, op. cit. 
ción más cercana a la perspectiva de desarrollo en los países, que de conflicto en el ámbito internacional. De este modo, se apreció como una herramienta para la creación de institucionalidad estatal, que pudiese establecer un control efectivo de los espacios territoriales. ${ }^{60}$ Incluso los postulados del ámbito internacional, desarrollados desde una base geopolítica, buscaban emplear recursos en pos de la integración de los países de la región, ofreciendo al mismo tiempo un contrapeso a las perspectivas hegemónicas de las grandes potencias, en especial en tiempos de la Guerra Fría. ${ }^{61}$

$\mathrm{Y}$ pese a que los contextos regionales e internacionales han variado considerablemente con respecto a lo vivido durante el conflicto bipolar, en el siglo xxi en la región aún se mantienen concepciones derivadas del pensamiento militar en la disciplina, demostrando así tanto la fuerte influencia que ha mantenido el ámbito castrense en esta rama del conocimiento, como también el hecho de que desde la misma academia no se ha hecho un esfuerzo para discutir las nuevas perspectivas teóricas y conceptuales de las nociones más contemporáneas, incluyendo lo planteado por la geopolítica crítica. ${ }^{62} \mathrm{Y}$, más aun, se destaca que en momentos en que el mundo repudiaba la geopolítica, producto de la remembranza de lo "nazi”, en la región se siguió desarrollando la disciplina, pero en su base epistémica positivista y teóricamente clásica. ${ }^{63}$

60 Andrés Rivarola, “Geopolitics of Integration' and the Imagination of South America”, Geopolitics, vol. 16, núm. 4 (2011), pp. 846-864; Gerardo Caetano, "De la "Suiza de América" al "Uruguay como problema”. La génesis del pensamiento de Alberto Methol Ferré”, Iberoamericana - Nordic Journal of Latin American and Caribbean Studies, vol. 47, núm. 1 (2018), pp. 63-73.

61 Therezinha de Castro, “Antártica - o assunto do momento", Boletim Geográfico, vol. 16, núm. 142 (1958), pp. 42-49.

${ }^{62}$ Lester Cabrera, "Geopolítica en América del Sur: desde la militarización de la disciplina a la necesidad del debate académico", Revista Chilena de Derecho y Ciencia Política, vol. 8, núm. 2, 2017, pp. 167-188.

${ }^{63}$ Detlef Nolte y Leslie Wehner, op. cit. 
Sin perjuicio de lo anterior, es posible rescatar algunas propuestas teóricas propias de la región, y que ayudan a enfatizar los problemas y realidades que, casi de manera exclusiva, vive Sudamérica en su totalidad, aunque con diferentes matices, pese a que en este punto es claro un desconocimiento o mal entendimiento de lo que comprende la geopolítica crítica o los parámetros más contemporáneos de la geopolítica. ${ }^{64}$ De esta manera, se destacan perspectivas que, desde una base epistémica reflectivista, plantean problemas como la protección de los recursos naturales regionales, la perspectiva imperial y hegemónica de ciertos Estados extrarregionales e, incluso, los desafíos de los modelos de integración regional. ${ }^{65}$

Producto de la incorporación de nuevos esquemas de pensamiento, principalmente derivados de las problemáticas regionales, se produce un nuevo campo sobre el cual es posible incorporar perspectivas más contemporáneas para la comprensión y análisis de los fenómenos, desde un enfoque más amplio. Éstos serían algunos de los factores y variables que la geopolítica crítica ayudaría a incorporar a las nociones de la geopolítica clásica propias de la región, considerando procesos como la globalización y un cambio de interpretación de los factores clásicos en la construcción de una institución moderna, como el Estado. Ello daría como resultado un conocimiento más holístico y amplio de los fenómenos que afectan Sudamérica.

${ }^{64}$ Luis González, "Organización del espacio global en la geopolítica 'clásica': una mirada desde la geopolítica crítica", Revista de Relaciones Internacionales, Estrategia y Seguridad, vol. 13, núm. 1 (2018), pp. 221-238; Francisco Le Dantec, "Contribución de la geopolítica crítica a la comprensión de la actual concepción de seguridad", Política y Estrategia, núm. 108 (2007), pp. 71-82.

${ }^{65}$ Mónica Bruckman, Recursos naturales y la geopolítica de la integración sudamericana, Quito, Instituto de Altos Estudios Nacionales, 2012; Atilio Borón, América Latina en la geopolítica del imperialismo, Buenos Aires, Luxemburgo, 2013; Miguel Ángel Barrios, "La geopolítica sudamericana del siglo xxi", Geopolitica y Estrategia Suramericana. Perspectivas académicas, Sangolquí, Centro de Estudios Estratégicos-ESPE, 2014, pp. 54-69. 
Pero la cuestión más relevante es que, como tal, la geopolítica aún no se encuentra realmente imbuida en una construcción de conocimiento, específicamente en el plano de los estudios internacionales en la región. Y este aspecto no es menor debido a que en esta rama de las ciencias sociales aún se sigue reflexionando sobre parámetros derivados del primer mundo, los cuales no se aplican necesariamente a la realidad regional. En este sentido, pese a que la geopolítica crítica también se deriva de una visión de pensamiento de otras latitudes, su uso e incorporación en los esquemas analíticos, como el caso del valor del discurso y los "códigos geopolíticos", ayudarían a reconocer los elementos que subyacen en iniciativas tanto nacionales como regionales, en torno a problemáticas puntuales y, con ello, comprender de mejor manera la realidad regional, siempre en términos geopolíticos.

\section{Conclusiones}

Por lo general, cuando se hace referencia a la geopolítica crítica, se realiza como una forma de expresión en contra de los preceptos de la geopolítica clásica, pero lo cierto es que ambas posiciones no necesariamente se consideran antagónicas e irreconciliables, sino que es factible establecer un grado de complementariedad, e incluso, de reforzamiento en la comprensión de los diversos postulados de cada una de las mencionadas ramas. Así, para entender de mejor forma los postulados clásicos, la geopolítica crítica establece un marco de análisis adecuado para ello. Incluso con la composición de una serie de lineamientos clásicos en geopolítica, si bien cumplen con las cualidades "negativas" que propugna y "descubre" la geopolítica crítica, también se observan aspectos desconocidos de ésta, que revitalizan y actualizan el conocimiento geopolítico. Y desde aquella visión, la geopolítica clásica complementa la visión crítica, al señalar la importancia de elementos como el territorio y la necesaria vinculación geográfica de los aspectos que se analizan. Con ello, no sola- 
mente se cumple el objetivo de presentar un desarrollo más complejo del concepto de geopolítica sino que, al mismo tiempo, se genera un límite para establecer lo que es y lo que no es geopolítica.

Las limitaciones de la geopolítica crítica, si bien se manifiestan incluso por los mismos autores que han desarrollado aquella disciplina, han generado una nueva visión en torno a los preceptos que son parte, en algunas ocasiones en condiciones de relevancia, del conocimiento geopolítico en general. La composición teórica de la geopolítica crítica se considera como tal debido a que, si bien identifica problemas, no propone soluciones, y lleva a un camino donde el desafío para proponer alguna mejora es justamente eso: proponer soluciones, pero con mayor congruencia metodológica.

Si lo anterior se aplica a la realidad regional, tomando en cuenta el grado de desarrollo de la geopolítica y los fenómenos o problemas que se muestran, la vinculación entre lo que implica "lo clásico" y "lo crítico" se vuelve necesaria. Sin embargo, para lograrlo, es imperativo comenzar a debatir sobre nuevas bases epistémicas y teóricas, que permitan la deconstrucción y discusión en torno a los parámetros clásicos que sostienen el conocimiento geopolítico regional. Sobre este punto, si bien se han establecido patrones relacionados, especialmente en lo relativo a fenómenos y procesos sociales, incluso con alcance internacional, se tiene el desafío de incorporar aquellos planteamientos sobre una base metodológica clara y explícita, con el fin de no caer en las debilidades mencionadas de la geopolítica crítica.

La región posee una fuerte vinculación con el conocimiento geopolítico, lo cual se manifiesta en sus políticas internas como externas. Pero los elementos intrínsecos del pensamiento geopolítico sudamericano deben actualizarse e incluso "democratizarse", tomando en cuenta que pese a estar en pleno siglo XXI, se siguen repitiendo patrones del siglo pasado, en particular el casi monopolio del ámbito castrense sobre la disciplina. Y ello también incluye masificar el con- 
cepto de geopolítica como tal, pero desde una base académica. La geopolítica crítica podría ayudar en esta labor, desde una óptica en la que los aspectos a discutir sean metodológica y teóricamente claros, específicos y acordes con los problemas de la región.

\section{Bibliografía}

Agnew, John y Stuart Corbridge, Mastering Space. Hegemony, territory and international political economy, Londres, Routledge, 1995.

Agnew, John, Geopolitics: Re-visioning World Politics, Londres, Routledge, 2003.

Atencio, Jorge, ¿Qué es la geopolítica?, Buenos Aires, Editorial Pleamar, 1968.

Atkinson, David y Klaus DodDs, "Introduction to geopolitical traditions: a century of geopolitical thought", en Klaus Dodds y David Atkinson (eds.), Geopolitical Traditions. A century of geopolitical thought, Nueva York, Routledge, 2000, pp. 1-25.

Barton, Jonathan, A Political Geography of Latin America, Nueva York, Routledge, 1997.

BArrios, Miguel Ángel, "La geopolítica sudamericana del siglo xxi”, Geopolítica y Estrategia Suramericana. Perspectivas académicas, Sangolquí, Centro de Estudios Estratégicos-ESPE, 2014, pp. 54-69.

Borón, Atilio, América Latina en la geopolitica del imperialismo, Buenos Aires, Luxemburgo, 2013.

Bruckman, Mónica, Recursos naturales y la geopolítica de la integración sudamericana, Quito, Instituto de Altos Estudios Nacionales, 2012.

Cabrera, Lester, "Geopolítica en América del Sur: desde la militarización de la disciplina a la necesidad del debate académico", Revista Chilena de Derecho y Ciencia Política, vol. 8, núm. 2, 2017, pp. 167-188.

CABRERA, Lester, "La vinculación entre geopolítica y seguridad: algunas apreciaciones conceptuales y teóricas", URVIO, Revista 
Latinoamericana de Estudios de Seguridad, núm. 20, 2017, pp. 111-125.

Caetano, Gerardo, "De la 'Suiza de América' al "Uruguay como problema”. La génesis del pensamiento de Alberto Methol Ferré", Iberoamericana - Nordic Journal of Latin American and Caribbean Studies, vol. 47, núm. 1, 2018, pp. 63-73.

Cairo, Heriberto, "Re-pensando la geopolítica: la renovación de la disciplina y las aportaciones de John A. Agnew" (Prólogo), en John Agnew, Geopolítica. Una re-visión de la política mundial, Madrid, Trama Editorial, 2005, pp. 9-16.

Castro, Therezinha de, "Antártica - o assunto do momento", Boletim Geográfico, vol. 16, núm. 142, 1958, pp. 42-49.

Child, John, "Geopolitical Thinking in Latin America”, Latin American Research Review, núm. 14, vol. 2, 1979, pp. 89-111.

Cohen, Saul, Geopolitics. The Geography of International Relations, Nueva York, Rownman \& Littlefield, 2015.

Dahlman, Carl, "Geopolitics", en Carolyn Gallaher et. al. (eds.), Key Concepts in Political Geography, Londres, SAgE, 2009, pp. 87-98.

Dalby, Simon, Creating the Second Cold War. The Discourse of Politics, Londres, Pinter Publishers, 1990.

Dalby, Simon, "Imperialism, Domination, Culture: The Continued Relevance of Critical Geopolitics", Geopolitics, vol. 13, núm. 3, 2008, pp. 413-436.

DodDs, Klaus, Global Geopolitics. A Critical Introduction, Londres, Pearson Prentice Hall, 2005.

DodDs, Klaus, Geopolitics: A Very Short Introduction, Nueva York, Oxford University Press, 2007.

DodDs, Klaus, Merje Kuus y Joanne Sharp, "Introduction: Geopolitics and its Critics", en Klaus Dodds, Merje Kuus y Joanne Sharp (eds.), The Ashgate Research Companion to Critical Geopolitics, Londres, Ashgate Publishing, 2013, pp. 1-14.

Flint, Colin, Introduction to Geopolitics, Londres, Routledge, 2006.

GonzÁlez, Luis, "Organización del espacio global en la geopolítica 'clásica': una mirada desde la geopolítica crítica”, Revista de Relaciones Internacionales, Estrategia y Seguridad, vol. 13, núm. 1, 2018, 221-238. 
Graham, Stephen, "Introduction: Cities, Warfare, and States of Emergency", en Stephen Graham (ed.), Cities, War, and Terrorism. Towards an Urban Geopolitics, Nueva York, Blackwell Publishing, 2004, pp. 1-26.

Haverluk, Terrence, Kevin Beauchemin y Brandon Mueller, "The Three Critical Flaws of Critical Geopolitics: Towards a Neo-Classical Geopolitics”, Geopolitics, núm. 19, 2014, pp. 19-39. Hepple, Leslie, "South American Heartland: The Charcas, Latin American Geopolitics and Global Strategies", The Geographical Journal, vol. 170, núm. 4, 2004, pp. 359-367.

Kearns, Gerry, "Imperial Geopolitics", en John Agnew, Katharyne Mitchell y Gearoid O'Tuathail (eds.), A Companion to Political Geography, Nueva York, Blackwell Publishing, 2003, pp. 173-186. Kelly, Phil, “A Critique of Critical Geopolitics”, Geopolitics, vol. 11, 2006, pp. 24-53.

Kelly, Phil, Classical Geopolitics. A New Analytical Model, Stanford, University Press, 2016.

Lacoste, Yves, "Las etapas de la geopolítica", en Leopoldo González Aguayo (edit.), Cuaderno de Trabajo. Antología. Los principales autores de las escuelas de la geopolítica en el mundo, Ciudad de México, UNAM, 2011, pp. 11-22.

Lacoste, Yves, Geopolitica. La larga historia de hoy, Madrid, Síntesis, 2008.

Le Dantec, Francisco, "Contribución de la geopolítica crítica a la comprensión de la actual concepción de seguridad”, Política y Estrategia, núm. 108, 2007, pp. 71-82.

Mierelles, Carlos, Antología geopolítica de autores militares chilenos, Santiago, Centro de Estudios e Investigaciones Militares, 2000. Mountz, Alison, "The Other”, en Carolyn Gallaher et. al. (eds.), Key Concepts in Political Geography, Londres, sAge, 2009, pp. 328-338.

MüLLER, Martin, "Reconsidering the concept of discourse for the field of critical geopolitics: Towards discourse as language and practice”, Political Geography, núm. 27, 2008, pp. 322-338.

Natter, Wolfgang, "Geopolitics in Germany, 1919-1945", en John Agnew, Katharyne Mitchell y Gearoid O’Tuathail (eds.), 
A Companion to Political Geography, Nueva York, Blackwell Publishing, 2003, pp. 187-203.

Nogué, Joan y Joan Rufrí, Geopolítica, Identidad y Globalización, Barcelona, Ariel Geografía, 2001.

Nolte, Detlef y Leslie Wehner, "Geopolitics in Latin America, Old and New", en David Mares y Arie Kacowicz (eds.), Routledge Handbook of Latin American Security, Nueva York, Routledge, 2016, pp. 33-43.

NunN, Fredercik, Relaciones militares civiles sudamericanas en el siglo XXI. Sombras del pasado y formas de lo que vendrá, Santiago, Academia de Guerra del Ejército de Chile, 2011.

O'Tuathail, Gearoid y John Agnew, "Geopolitics and discourse: Practical geopolitical reasoning in American foreign policy", Political Geography, vol. 11, 1992, pp. 190-204.

O'Tuathail, Gearoid, Critical Geopolitics. The Politics of Writing Global Space, Londres, Routledge, 1996.

O'Tuathail, Gearoid y Simon Dalby, "Introduction: Rethinking geopolitics: towards a critical geopolitics", en Gearoid O'Tuathail y Simon Dalby (eds.), Rethinking Geopolitics, Londres, Routledge, 1998, pp. 1-15.

O'Tuathail, Gearoid, "Postmodern geopolitics? The modern geopolitical imagination and beyond", en Gearoid O'Tuathail y Simon Dalby (eds.), Rethinking Geopolitics, Londres, Routledge, 1998, pp. 16-38.

O'Tuathail, Gearoid, "Thinking Critically about Geopolitics", en Gearoid O'Tuathail, Simon Dalby y Paul Routledge (eds.), The Geopolitics Reader, Londres, Routledge, 2006, pp. 1-14.

Rivarola, Andrés, “'Geopolitics of Integration' and the Imagination of South America”, Geopolitics, vol. 16, núm. 4, 2011, pp. 846-864.

Routledge, Paul, "Introduction: Anti-Geopolitics", en Gearoid O'Tuathail, Simon Dalby y Paul Routledge (eds.), The Geopolitics Reader, Londres, Routledge, 2006, pp. 245-255.

SlOAN, Geoffrey y Colin GraY, "Why Geopolitics?", Journal of Strategic Studies, vol. 22, 1999, pp. 1-11.

TAYlor, Peter, "Geopolitics, Political Geography and Social Science”, en Klaus Dodds y David Atkinson (eds.), Geopolitical Tradi- 
tions. A century of geopolitical thought, Nueva York, Routledge, 2000, pp. 375-379.

Taylor, Peter y Colin Flint, Geografía política. Economía mundo, Estado-nación y localidad, Madrid, Trama Editorial, 2002.

Wallerstein, Immanuel, Geopolitica y Geocultura. Ensayos sobre el moderno sistema mundial, Barcelona, Editorial Kairós, 2011. 
